\title{
基准物质的性状对标准溶液标定的影响
}

-以硼砂 为例

刘莎莎 ${ }^{*}$, 李会香, 雷杰

复旦大学化学系, 上海 200433

摘要: 考查了酸碱滴定分析中的基准物质嗍砂的性状对 $\mathrm{HCl}$ 标准溶液标定的影响。对属同一批次但性状不同的两种硣 砂进行 $\mathrm{HCl}$ 标准溶液的标定, 所得的盐酸浓度平均值分别为 0.1049 和 $0.1052 \mathrm{~mol} \cdot \mathrm{L}^{-1}$, 随后将分析测量数据进行了显 著性检验, 表明二者存在显著性差异, 说明嗍砂的性状不同可能是由于其性质的部分改变而引起的, 这种性状的不同对 $\mathrm{HCl}$ 标准溶液浓度标定, 乃至后续未知酸碱浓度测定等数据的准确度均会造成较大的影响。

关键词：酸碱滴定；基准物质；硼砂；显著性检验

中图分类号：G64；O65

\section{Study on the Effect of Appearance of the Primary Standard on Standardizing the Standard Solution: Borax as an Example}

Shasha Liu *, Huixiang Li, Jie Lei

Department of Chemistry, Fudan University, Shanghai 200433, China.

Abstract: Borax in different appearance is compared and studied in standardization of the standard hydrochloric acid as primary standard. The obtained concentrations of hydrochloric acid are $0.1049 \mathrm{~mol} \cdot \mathrm{L}^{-1}$ and $0.1052 \mathrm{~mol} \cdot \mathrm{L}^{-1}$, respectively. Then based on the statistical test of the data, it was revealed that there are significant differences between the two. Additionally, appearance differences of borax, which may be caused by the change of its properties, will give rise to the error of subsequent measurements.

Key Words: Acid-base titration; Primary standard; Borax; Test of significance

分析化学是化学学科的重要分支, 也是科学研究中对物质进行定性、定量和结构分析的重要手 段和方法。通常我们将分析化学方法分为化学分析和仪器分析。滴定分析(也称容量分析), 是化学分 析中极其重要和经典的定量分析技术之一，是分析化学的基础，具有非常悠久的历史 ${ }^{[1,2]}$ 。

滴定分析法是建立在化学反应平衡基础上, 用标准溶液对待测体系进行滴定以测定物质含量的 分析方法, 其操作简便、快速, 最突出的优势是分析准确度高, 具有较大的实用价值。滴定分析中 的四大滴定方法(即酸碱滴定、络合滴定、氧化还原滴定和沉淀滴定), 一直以来都是分析化学理论教 学和实验教学中的基础内容, 且比重较大 ${ }^{[3-5]}$ 。标准溶液是具有准确浓度的溶液, 可通过准确称取基 准物质直接配制, 也可通过间接法标定(即先配制接近所需浓度的溶液, 然后准确称取基准物质, 用 滴定的方法测定所配溶液的准确浓度)得到。基准物质是指能够直接用于配制标准溶液或标定溶液浓

收稿: 2020-01-26; 录用: 2020-03-08; 网络发表: 2020-03-17

“通讯作者, Email: ssliu@fudan.edu.cn

基金资助: 复旦大学 2019 年度第二批教学研究与改革实践项目(2019A011) 
度的物质, 在滴定分析中基准物质的选用标准为: 纯度高; 组成与化学式精确匹配; 性质稳定, 不 易与大气中组分发生反应、不易吸潮、不易丢失结晶水, 且需要有特定的前处理和保存方法, 以确 保性质不发生变化; 符合滴定分析对化学反应的要求; 尽可能有较大的摩尔质量, 以减小称量的相 对误差 ${ }^{[1]}$ 。

本文主要以酸碱滴定分析中 $\mathrm{HCl}$ 标准溶液的标定为例, 探讨基准物质嗍砂对标准溶液浓度标定 结果的影响, 为参与滴定分析实验教学的技术人员在教学实验准备过程中对硼砂基准物质的选用提 供一个直观便利的参考方法。

\section{1 酸碱滴定分析基准物质的选用}

酸碱滴定法是以酸碱中和反应为基础的滴定分析方法, 也是滴定分析实验中进行基本操作训练 的先导实验。在酸碱滴定分析中, 标定 $\mathrm{HCl}$ 标准溶液常用的基准物质有无水碳酸钠 $\left(\mathrm{Na}_{2} \mathrm{CO}_{3}\right)$ 和嗍砂。 因为 $\mathrm{Na}_{2} \mathrm{CO}_{3}$ 容易吸收空气中的水分和二氧化碳, 所以 $\mathrm{Na}_{2} \mathrm{CO}_{3}$ 在使用前需要在 $270-300{ }^{\circ} \mathrm{C}$ 烘至恒 重后于干燥器内保存; 硼砂在相对湿度约 39\%-99\% $\left(20{ }^{\circ} \mathrm{C}\right)$ 时很稳定, 但当相对湿度降至 39\%以下 时会部分风化, 因此嗍砂须置于含有氯化钠和蔗糖饱和溶液的恒湿器(相对湿度约 $60 \%$ )中 ${ }^{[1,6-8]}$ 。

用 $\mathrm{Na}_{2} \mathrm{CO}_{3}$ 作基准物质标定 $\mathrm{HCl}$ 标准溶液时, 以甲基橙作指示剂, 终点变色不够敏锐 ${ }^{[9-11]}$; 同 时, 由于 $\mathrm{Na}_{2} \mathrm{CO}_{3}$ 摩尔质量小, 称量误差会被放大 ${ }^{[7]}$; 并且鉴于教学对象均为初次接触较为完整和正 统的滴定分析体系, 对指示剂变色的实际体验几乎为零, 故为了在基础的酸碱滴定分析中培养学生 的正确滴定操作以减少后续教学实验中的误差, 和逐步训练对指示剂的变色敏感度, 本学科实验课 程项目中选用摩尔质量较大的硼砂作为基准物质对 $\mathrm{HCl}$ 标准溶液进行标定。

\section{2 在基准物质前处理和保存过程中发现的问题}

由于嗍砂作为基准物质, 需要保存在含有氯化钠和蔗糖饱和溶液的恒湿器中, 化学分析实验室 的实验技术人员在前一学期结束即将硼砂请购入库, 并保存在恒湿器内平衡 2-3 个月, 直至下一学 期实验当天为学生分装于称量瓶内备用。由于每学期的酸碱滴定实验涉及的人数较多, 以复旦大学 为例, 包括化学系、高分子科学系、材料科学系和生命科学学院等专业的学生和老师, 共计约 300 人次, 因此, 所需要准备的硼砂基准物质用量很大, 考虑到硼砂来源的一致性, 我们采用的是分析 纯的瓶装硼砂, 实验中需要保证样本无差异。

在实验准备过程中, 我们发现来源于同一厂家, 批号相同的硼砂, 性状有所不同, 如图 1 所示。 1 \#硼砂呈细小的颗粒状, 颗粒分明, 如同散沙一般; 而 2 \#硼砂则呈细粉状, 颗粒更细小, 如同面粉 一般。2\#硼砂在恒湿器内放置一段时间后容易结块, 为了使硼砂在恒湿器中平衡均匀, 需要频繁地 将其结块搅散, 以期在不同教学周也能保持一致, 保证学生数据的连贯性。
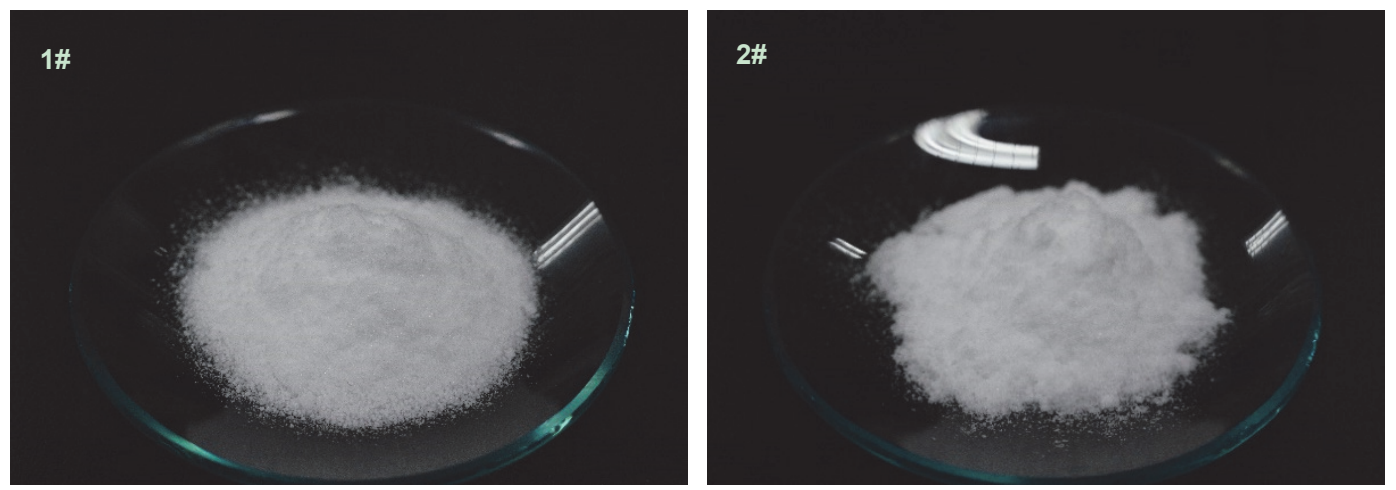

图 1 来自同一批次不同性状的嗍砂 
按照老一辈技术人员的传统经验, 属于同一批次的嗍砂在一定程度上默认为无差别, 对学生数 据基本无影响。然而基于科学严谨的学术态度, 我们秉持着科学研究也需要敢于怀疑的理念, 通过 实验对这两种性状的硼砂进行了研究, 探讨它们对盐酸溶液的标定以及学生测定未知酸的数据准确 度的影响, 考查两种嗍砂是否存在显著性差异。

\section{3 不同性状的嗍砂对 $\mathrm{HCl}$ 标准溶液标定的影响}

\section{1 实验试剂}

$\mathrm{HCl}$ 标准溶液： $0.1 \mathrm{~mol} \cdot \mathrm{L}^{-1}$, 待标定; 甲基红指示剂: $0.2 \%$ 乙醇溶液; 嗍砂：保存于恒湿器中。 实验中所用试剂均为分析纯, 购自国药集团化学试剂(沪试)有限公司。

\section{2 实验步骤}

(1) 以 1 \#硼砂作为基准物质标定 $\mathrm{HCl}$ 标准溶液。

准确称取 0.5-0.6 g 1\#硼砂 10 份, 分别置于干净的雉形瓶中, 加入 $50 \mathrm{~mL}$ 水溶解, 加入甲基红 指示剂 2 滴, 以 $0.1 \mathrm{~mol} \cdot \mathrm{L}^{-1}$ 的 $\mathrm{HCl}$ 标准溶液滴定至黄色变为橙红色, 即为终点。

(2) 以 2 \#硼砂作为基准物质标定 $\mathrm{HCl}$ 标准溶液。

准确称取 0.5-0.6 g 2\#硼砂 10 份, 分别置于干净的雉形瓶中, 加入 $50 \mathrm{~mL}$ 水溶解, 加入甲基红 指示剂 2 滴, 以 $0.1 \mathrm{~mol} \cdot \mathrm{L}^{-1}$ 的 $\mathrm{HCl}$ 标准溶液滴定至黄色变为橙红色, 即为终点。

\section{3 结果与讨论}

由同一技术人员进行称量和滴定操作, 以减少因分析人员造成的主观误差, 通过两组滴定, 分 别得到 $\mathrm{HCl}$ 标准溶液的浓度, 见表 1 。经格拉布斯(Grubbs)检验法判断, 95\%置信水平下, 可以认为 表 1 中 1 \#硼砂数据中的 0.1052 为异常值, 应舍去, 故后面的计算中均舍弃此值 ${ }^{[1]}$ 。需要说明的是, 平均值、标准偏差等的计算, 均用 Excel 中的相关参数从原始测量数据一步计算得到, 不做中间计 算值的有效数字修约。下同。

表 1 以嗍砂作为基准物质标定所得的 $\mathrm{HCl}$ 标准溶液的浓度

\begin{tabular}{|c|c|c|c|}
\hline \multicolumn{2}{|c|}{$1 \#$} & \multicolumn{2}{|c|}{$2 \#$} \\
\hline 样品号 & 浓度/(mol· $\left.\mathrm{L}^{-1}\right)$ & 样品号 & 浓度 $/\left(\mathrm{mol} \cdot \mathrm{L}^{-1}\right)$ \\
\hline 1 & 0.1049 & 1 & 0.1052 \\
\hline 2 & 0.1049 & 2 & 0.1052 \\
\hline 3 & 0.1049 & 3 & 0.1051 \\
\hline 4 & 0.1048 & 4 & 0.1053 \\
\hline 5 & 0.1049 & 5 & 0.1052 \\
\hline 6 & 0.1049 & 6 & 0.1052 \\
\hline 7 & 0.1048 & 7 & 0.1052 \\
\hline 8 & 0.1049 & 8 & 0.1052 \\
\hline 9 & 0.1052 & 9 & 0.1052 \\
\hline 10 & 0.1049 & 10 & 0.1053 \\
\hline 平均值(除去 9 号样) & 0.1049 & 平均值 & 0.1052 \\
\hline 标准偏差 $s$ (除去 9 号样) & $0.045 \%$ & 标准偏差 $s$ & $0.057 \%$ \\
\hline
\end{tabular}

通常来说, 对于总体方差 $\sigma^{2}$ 未知的两组数据, 采用 $F$ 检验和 $t$ 检验来验证两者有无显著性差 异。 $F$ 检验是对样本方差的比较, 主要用于说明两组数据的精密度有无显著性差异; 而 $t$ 检验则是 用于说明两组数据的平均值有无显著性差异。对于 1 \#嗍砂和 2 \#硼砂这两组数据进行 $F$ 检验, 计算 
得到 $F=1.66$, 小于查表所得 $F_{0.025 /(9,8)}=4.36$ (单侧) ${ }^{[12]}$, 说明有 $95 \%$ 的把握认为 $1 \#$ 硼砂和 $2 \#$ 硼砂所 得两组数据的精密度无显著性差异。计算两组数据的合并标准偏差后, 再进行 $t$ 检验, 计算得到 $t=$ 14.2 , 大于查表所得值 $t_{0.025,17}=2.11$ (单侧) ${ }^{[12]}$, 故有 $95 \%$ 的把握认为 1 \#硼砂和 2 \#硼砂有显著性差 异。

为了对比, 我们将购于同月, 但是不同批次, 性状与 $2 \#$ 硼砂相同的硼砂 (定为 $3 \#)$, 也进行了 $\mathrm{HCl}$ 标准溶液的标定实验, 所得的 $\mathrm{HCl}$ 标准溶液的浓度平均值为 $0.1052 \mathrm{~mol} \cdot \mathrm{L}^{-1}(n=10, s=0.043 \%)$,

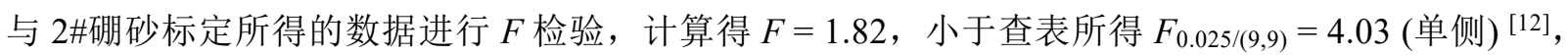
因此有 $95 \%$ 的把握认为这两组数据的精密度无显著性差异; 虽然保留四位有效数字时, 采用 2 \#嗍砂 与 3 \#硼砂标定所得到的两组数据的平均值相等, 但是不进行有效数字修约, 采用原始数据时仍可进 行 $t$ 检验, 计算得 $t$ 值为 1.35 , 小于查表所得值 $t_{0.025,18}=2.10$ (单侧) ${ }^{[12]}$, 故有 $95 \%$ 的把握认为 $2 \#$ 硼 砂和 $3 \#$ 硼砂无显著性差异。

\section{4 总结}

本文以不同性状的嗍砂作为基准物质, 探讨其对 $\mathrm{HCl}$ 标准溶液标定结果的影响。首先, 以同一 批次的嗍砂作为研究对象, 分别以两种性状不同(1\#为细小颗粒状, 2\#为细粉状)的嗍砂作为基准物 质, 来标定 $\mathrm{HCl}$ 标准溶液, 得到标准溶液的浓度平均值分别为 0.1049 和 $0.1052 \mathrm{~mol} \cdot \mathrm{L}^{-1}$, 通过 $F$ 检 验和 $t$ 检验, 表明 1 \#和 2 \#两种硼砂存在显著性差异, 说明硼砂性状不同会对实验教学过程中后续测 定未知酸碱浓度数据的准确度产生显著影响。同样, 我们也对相同性状, 但属不同批次的两种嗍砂 (2\#和 3\#) 进行了标定数据的比较, 二者标定所得的 $\mathrm{HCl}$ 标准溶液浓度均为 $0.1052 \mathrm{~mol} \cdot \mathrm{L}^{-1}$, 同样通过 $F$ 检验和 $t$ 检验发现二者不存在显著性差异。由此说明即使是不同批次的硼砂, 如果性状相同, 仍 有可能不存在显著性差异。综上, 在教学实验准备过程中, 我们可以通过观察性状来对喼砂进行初 步的区分, 避免造成带教老师预做实验和学生教学实验的数据差异, 也可以为教辅工作节省出时间, 更加有效地进行实验室管理及教学改革等相关工作。

\section{参 考 文 献}

[1] 吴性良, 孔继烈. 分析化学原理. 第 2版. 北京: 化学工业出版社, 2010: 1-54.

[2] 赵滨, 马林, 沈建中, 卫景德. 无机化学与化学分析实验. 上海: 复旦大学出版社, 2019: 1-45.

[3] 柳玉英, 王平, 刘青, 萄红桃. 大学化学, 2016, 31 (9), 43.

[4] 方苗利. 教育现代化, 2017, No. 13, 11.

[5] 雷良萍, 龙文清. 大学化学, 2006, 21 (1), 55.

[6] 北京大学化学与分子工程学院分析化学教学组. 基础分析化学实验. 第 3 版. 北京: 北京大学出版社. 2010: 7-20.

[7] 赵红艳, 钟银飞, 祝红蕾, 郭佳佳. 现代测量与实验室管理, 2015, No. 1, 32.

[8] 夏玉宇. 化学实验室手册. 第 3 版. 北京: 化学工业出版社. 2015: 554-570.

[9] 付平. 计量技术, 1995, No. 9, 31 .

[10] 谢鹏. 广东轻工职业技术学院学报, 2012, 11 (3), 42 .

[11] 胡刚. 炼油与化工, 2014, 25 (2), 34.

[12] 陈平. 应用数理统计. 北京: 机械工业出版社, 2008: 371-377. 